\title{
NUMERICAL COMPUTATHON OF DYNAMIC STRESS INTENSITY FACTORS \\ BY A LAGRANGIAN FINITE-DIFFERENCE METHOD \\ (THE HEMP CODE)
}

Y. M. Chen

April 2, 1.974

Prepared for U.S. Atonic Energy Commission under contract No. W-7405-Eng-48 
MOTXE

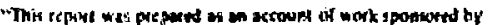

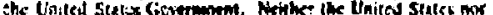

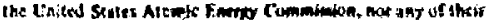

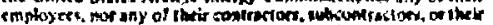

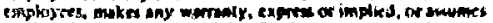

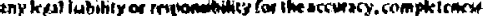

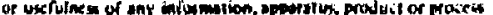

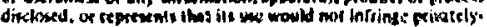
owned piphis."

Printed in the United States of America Available from

National Techniral Information Serviee

U, S. Department of Commerce 5285 Port Royal kioad Springfield, Virginia 22151

Price: Printed Copy \$ $\$$; Microfiche $\$ 0.95$

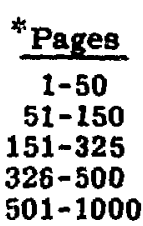

NTIS

Selling Frice

$\$ 4.00$

$\$ 5.45$

$\$ 7.60$

$\$ 10.60$

$\$ 13.60$ 
TID -4500 , UC -32

Mathematics

and Computers

\title{
노 \\ LAWRENCE LMEAMORE LAEOAATOAY \\ untwertyol Cattomia Lhemora Corfornin 94550
}

UCRL-51563

\section{NUMERICAL COMPUTATION OF OYNAMIC STRESS INTENSITY FACTORS \\ BY A LAGRANGIAN FINHTE-DIFFERENCE METHOD (THE HEMP CODE)}

\author{
x. M. Chen
}

MS. date: April 2, 1974

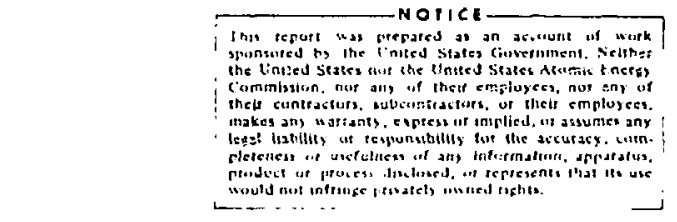

Permanent address: Department of Applied Mathematirs and Statistics

State University of New York at Stony Brook

Stony Brook, N. Y. 11790 


\title{
NUMERICAL COMPUTATION OF DYNAMIC STRESS INTENSITY FACTORS BY A LAGRANGIAN FINITE-DIFFERENCE METHOD (THE HEMP CODE)
}

\begin{abstract}
Numerical computation of the dynamic stress intensity factor for a centrally cracked rectangular bar, subjected tw uniaxial tensions in plane strain geometry with Heaviside-function time dependence, is carried out using the timedependent Lagrangian finite-difference code, HEMP. Excellent agreement is

found with results obtained from other methods. This demonstrates the capability and the rellability of the HEMP code as an extremely useful numerical method for solving dynamic problems in fractur: mechanics. Also discussed is the dynamic behavior of the stress intenglty factor and crack opening.
\end{abstract}

\section{Introduction}

The knowledge of the stress field in the vicinity of a crack is a pre requisite to the prediction of fracture and failure of the material. In order for the crack to propagate, the stress at the crack tip must exceed some critical value. For brittle materials, the linear elasticity model usually gives a reasonably good description of the macroscopic phenom ena of fracture in solids. However, for this model it is well known that regardless of the magnitude of the applied load the stress at the tip of a crack may have a singularity of $O\left(r^{-1 / 2}\right)$, where $r$ is the radial distance from the crack tip to the point of observation. ${ }^{1-4}$ To overcome the analysis difficulty that an infinite stress assumption causes, the stresses in the neighborhood of the crack tip are normalize 1 by rivltiplying them by a funition $O\left(r^{1 / 2}\right)$, yielding zero at the crack tip. This leads to the definition of the stress intensity factors. ${ }^{3}$ For dynamic problems, the dynamic stress intensity factor is defined in the same manner as for the static case. The only difference is that the dynamic stress intensity factor is a function of time ?.

Recently, considerable research has been done on the determination of the dyramic stress fields around a finite crack in an infinite elastic solid. $5-15$ However, the quasi-numerical methods used are incapable of solving dynamic problems with finite boundaries. On the other hand, the finite element method can be used to solve problems in fracture mechanics with finite boundaries provides 
the loads are static, ${ }^{16-20}$ Actually, finite element methods can be used for dynamic problems by applying a series of incremental static loads to approximate time variations. ${ }^{21-23}$ This approach requires inordinate amounts of computer time for two- and three-aimensional problems when a large number of elements are used, because a large set of simultaneous linear algebraic equations must be solved at each time step. It becomes especially inefficient when large matrices occur as in calculations involving three space dimensions. The work presenied here is concerned with the solution of problems involving dynamic fracture with finite boundaries using finite difference methods, which avoid the difficulties of the finite element method.
The main mathematica' tocl used is a Lagrangian finite-difference computer program, the HEMP code. The general program can be used to solve problems in three space dimension and time. ${ }^{24-26}$ In this paper we demonstrate the capability and the reliability of the HEMP code for solving dynamic problems in fracture mechanics by applying it to a simple twodilmensional but nont rivial dynamic problem.

For simplicity, a centrally cracked rectangular bar in plane strain geometry is considered. A uniaxial tension with a Heavisidefunction time dependence is applied. The details of the example are given in the next section, and the numerical results are presented graphically in the third section. Finally, in the last section, a detailed discussion of the numerical results is given

\section{Example Problem}

Consider a rectangular bar with a centrally located crack shown in Fig. 1. It is loaded dynamically in the axial direc- tion by a uniform tension $P(t)$ with Heaviside-function time dependence (Fig. 2). The boundary conditions given

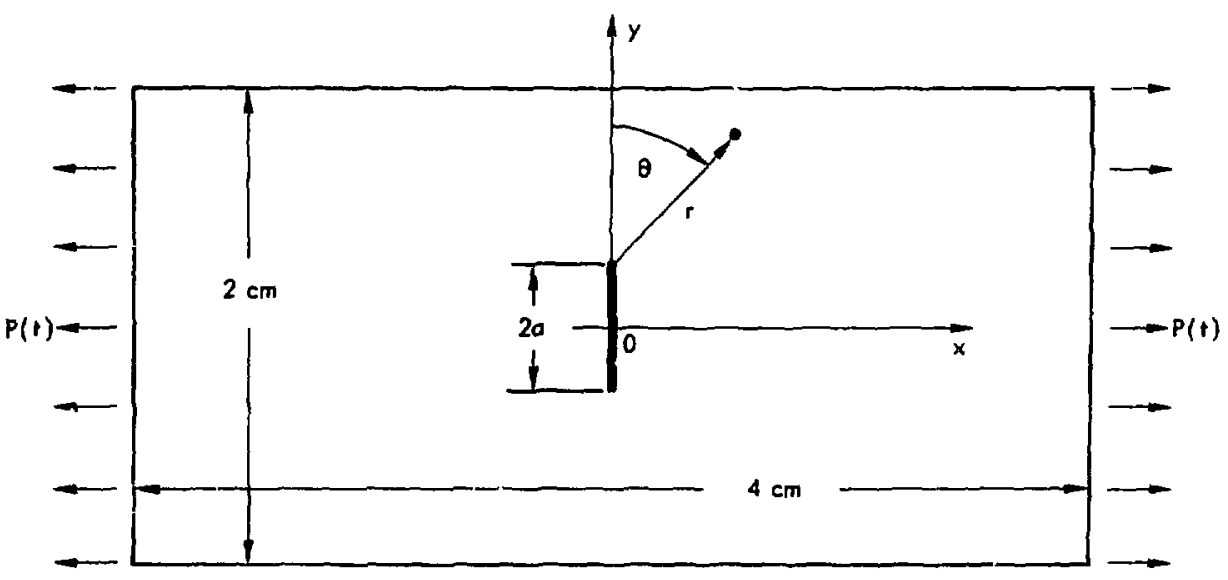

Fig. 1. Geometry of the bar in the example problem; $a=0.24 \mathrm{~cm}$. 


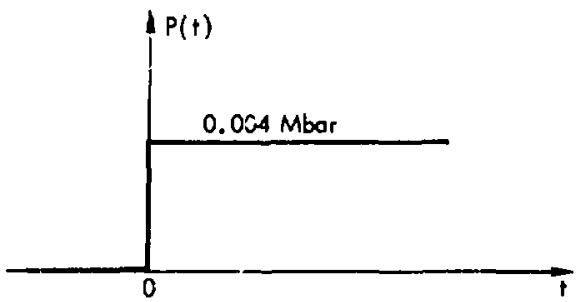

Fig. 2. Load curve assumed in example problem.

correspond to plane strain loading. The bar is issumed to be a linear elastic material with the following characteristics:

Shear modulus $G=0.76923$ Mbar,

Rulk modulus $\mathrm{K}=1.66667 \mathrm{Mbar}$,

Poisison's ratio $\nu=0.3$,

Density $\rho=5 \mathrm{~g} / \mathrm{cm}^{3}$.

Hence the longitudinal wave speed is

$v_{L}=\sqrt{\frac{K+\frac{4}{3} G}{\rho}} \approx 0.734 \mathrm{~cm} / \mu \mathrm{sec}$,

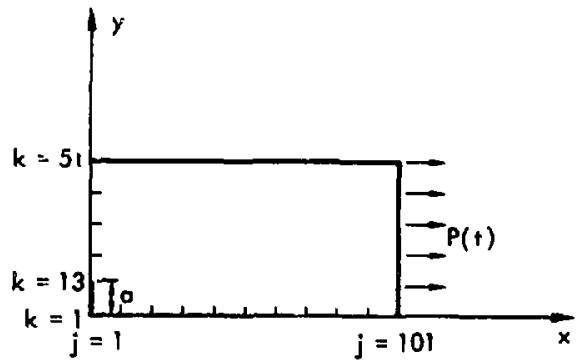

Fig. 3. Zoning of the quarter of the bar treated in the calculation.

the transverse wave speed is

$$
v_{T}=\sqrt{\frac{G}{\rho}} \approx 0.392 \mathrm{~cm} / \mu \mathrm{sec},
$$

and the Rayleigh wave speed ${ }^{27}$ is

$v_{R} \approx 0.495 v_{L} \approx 0.363 \mathrm{~cm} / \mu s e c$.

By symmetry it is sufficient to solve the quarter problem, as shown in Fig. 3. A grid of 50 zones/cm was used.

\section{Numerical Results}

Due to the inability of any finitedifference method to represent the extremely steep stress gradients that occur in the neighborhood of a crack tip, a subroutine for extrapolation of the mode I dynamic stress intensity factor $k_{1}(t)$ from the stress field in the vicinity of the crack tip is used here. The procedure uses the stress field equations in the neighborhood of the crack tip ( $r=0)$, aseuming an iniinite elastic medium,

$$
\sigma_{i m}(t) \leadsto \frac{k_{1}(t)}{(2 \pi r)^{1 / 2}} f_{i m}(\theta),
$$

where

$$
f_{x x}(\theta)=\left(1+\sin \frac{\theta}{2} \sin \frac{3 \theta}{2}\right) \cos \frac{\theta}{2}
$$$$
\mathrm{f}_{\mathrm{yy}}(\theta)=\left(1-\sin \frac{\theta}{2} \sin \frac{3 \theta}{2}\right) \cos \frac{\theta}{2},
$$

$f_{x y}(\theta)=f_{y x}(\theta)=\sin \frac{\theta}{2} \cos \frac{\theta}{2} \cos \frac{3 \theta}{2}$.

From (4) we define for every time step

$$
k_{1}^{*}(n \Delta t) \equiv \frac{\left(2 \pi r_{j k}\right)^{1 / 2}}{f_{i m}\left(\theta_{j k}\right)^{\prime}} \sigma_{i m}(n \Delta t) .
$$




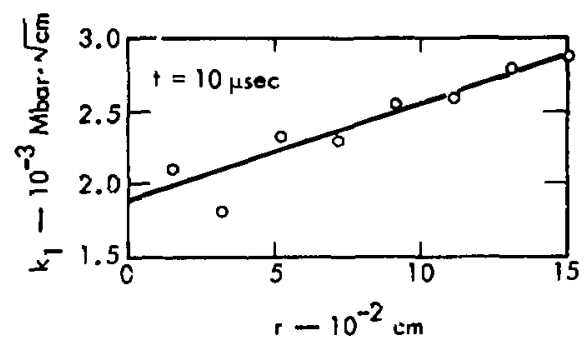

Here $\left(r_{j k}, \theta_{j k}\right)$ are the radial coordinates of a noint $(j, k)$ referred to the crack tip. Once the extrapolation path is chosen. Eq. (5) can be considered as a discrete functional relation between $k_{1}^{k}(n \Delta t)$ and

Fig. 4. A typical linear extrapolation curve for $k_{i}$.

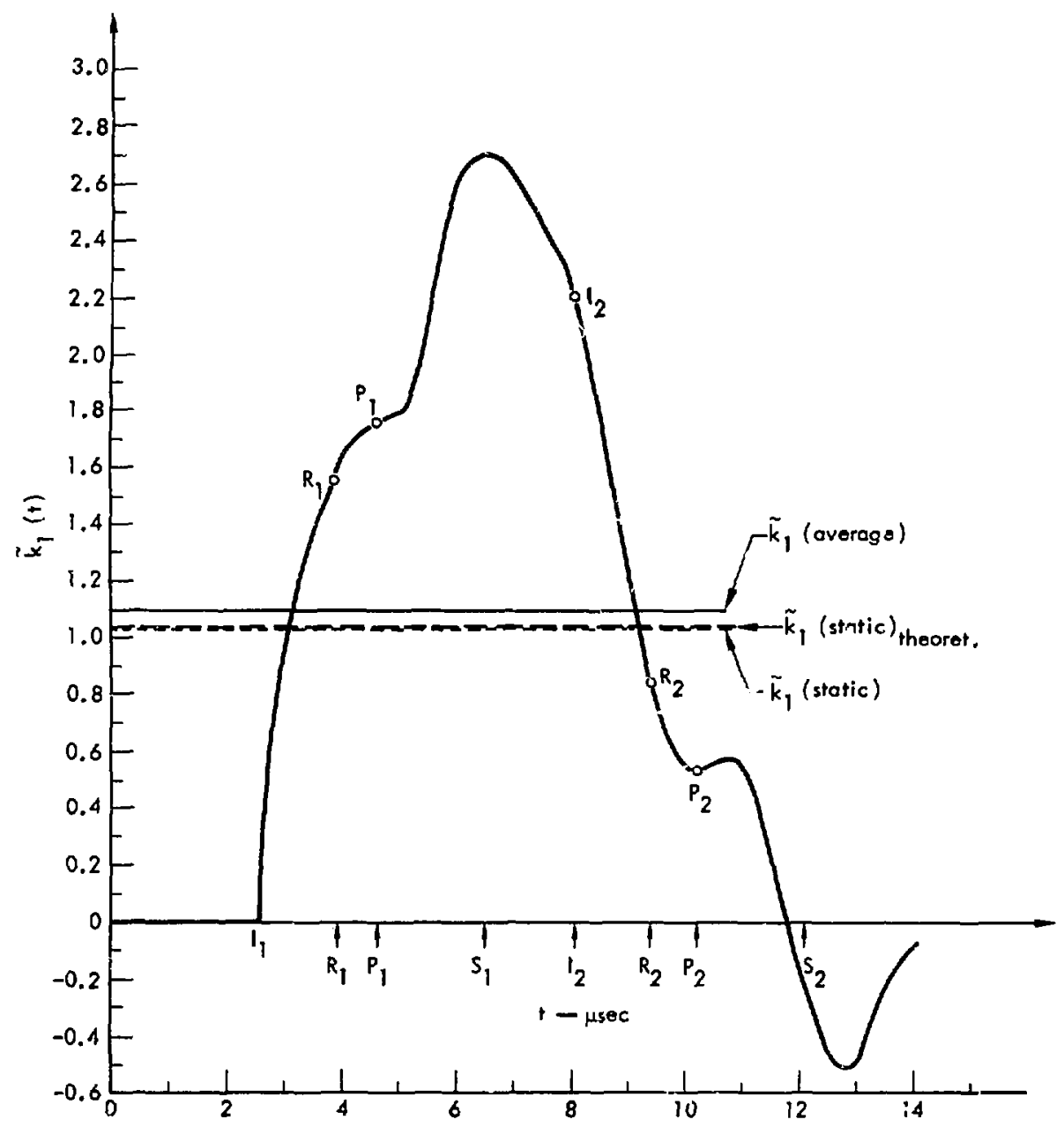

Fig. 5, Curve of $\tilde{k}_{1}\langle t\rangle$ vg $t$. 
$\mathbf{r}_{\mathrm{jk}}$. Our particular extrapolation path along the straight line $x=0.01 \mathrm{~cm}$ is the closest to the best path (see Appendix). Nexi, these discrete data are fitted by least-squares to a straight line,

$$
k_{1}^{*}(n \Delta t)=k_{1}(n \Delta t)+\beta(n \Delta t) r
$$

where $k_{1}(n \Delta t)$ is the approximate value of the mode $\mathrm{I}$ dymamic stress intensity factor at time nat. The error for this kind of straight-line extrapolation is $O\left(r^{2}\right)$ (see Aprendix). Note that the data for two to thrce zones neareat to the crack tip are discarded in the least-squares surve fitting. Extrapclations along lines in several directions were also made.

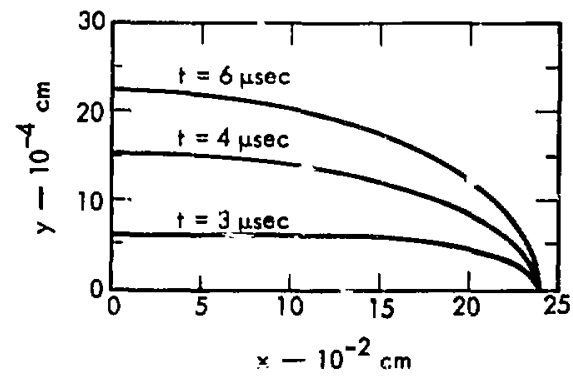

Fig. 6. Geometry of the crack opening at three different time steps.
The final values agreed to within $5 \%$. A typical plot of (s) and (6) is shown in Fig. 4. The normalized mode I dynamic stress intersity facto: $\tilde{k}_{1}(t)=k_{1}(t)$ / $P(t)(\pi a)^{1 / 2}$ is plotted against $t$ in $F(g .5$. Also, the geometry of the crack opening for several time steps is shown in Fig. 6. A $\sigma_{x x}$ contour plot is given in Fig. 7 . The above subroutine has been used successfully by others. ${ }^{17,28}$

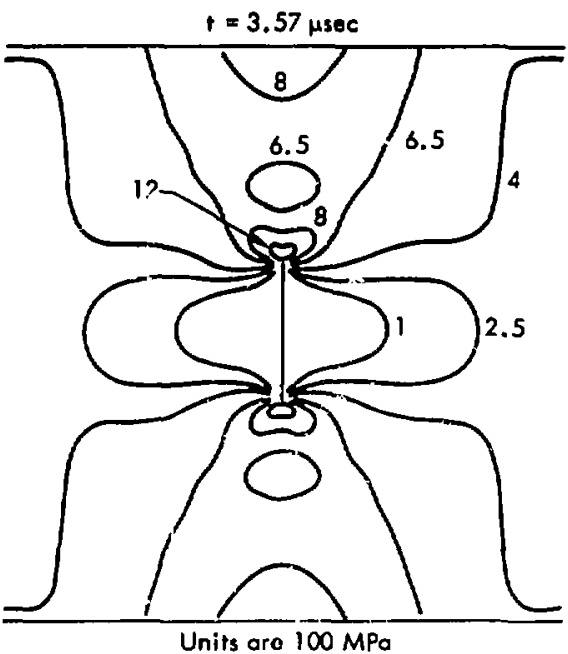

Fig. 7. A typical $\sigma_{x x}$ contour (retraced from computer plot).

\section{Discussion}

If there were no crack in the rectengular bar, the stress field in the bar due to the dynamic loading $P(t)$ of Fig. 2 would be a standing wave centered arounc' the appropriate static value. However, in the presence of the crack there are many oscillations in the $\tilde{K}_{1}(t)$-vs-t curve. These oscillations are due to scattering phenomena from the crack tip and the boundary surfaces.

On the time axis of Fig. 5, I, denotes the numerically computed (HEMP coie) time needed for the incident longitudinal wave to travel to the crack from the bar ends. Now, the following times are computed from the wave velocities, Eqs. (1), 
(2), and (3), and the true geometric dis tances: $\left(R_{1}-I_{1}\right)$ denctas the time needed for the Rayleigh wave, generated by the Initial incident waves, to travel between the two crack tips; $\left(P_{1}-I_{1}\right)$ de.lotes the time needed for the scattered longitudinal wave, generated by the initial incident waves, to tra .. from a crack tip to the nearest boundary surface of the bar and back to the same tip; similarly, $\left(S_{1}-I_{1}\right)$ corresponds to the scattered transverse wave; $I_{2}$ denotes the time needed for the incident longitudinal waves to travel the length of the bar, reflect from the boundary surface on the opposite side, and then travel back to the crack; analogous definitions are given to $\left(\mathrm{R}_{2}-\mathrm{I}_{2}\right),\left(\mathrm{P}_{2}-\mathrm{I}_{2}\right)$, and $\left(\mathrm{S}_{2}-\mathrm{I}_{2}\right)$ respectively, generated by these secondly incident longitudinal waves. Hence, with the help of these time marks one can identify the oscillations in the $\tilde{k}_{1}(t)-v s-t$ curve as being caused by the cancellation and the reinforcement of the incident waves by various scattered waves. The $\sigma_{x x}-v s-t$ curve (Fig. 8 ) at

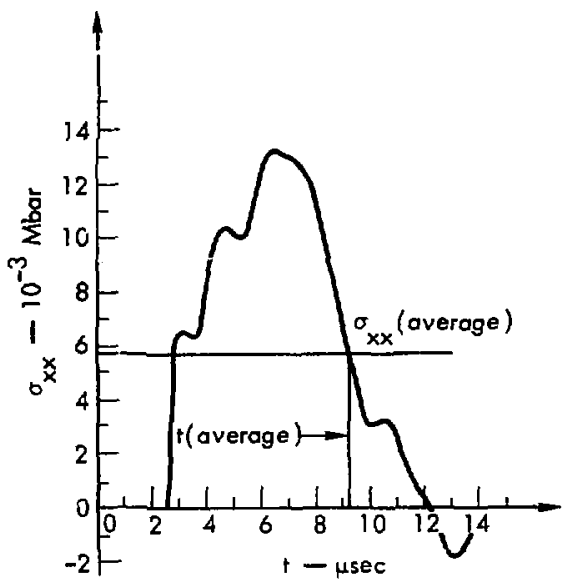

Fig. 8. The $\sigma_{x x}-v s-t$ curve at $(x, y)$ $=(0.01 \mathrm{~cm}, 0.37 \mathrm{~cm})$. $(x, y)=(0.01 \mathrm{~cm}, 0.37 \mathrm{~cm})$ has the same general behavior as the $\tilde{k}_{1}-v s-t$ curve except for the extra peak at $t \approx 3 \mu \mathrm{sec}$ due to the scattered longitudinal wave iraveling directly from the nearest crack tip to the point $(0.01 \mathrm{~cm}, 0.37 \mathrm{~cm})$.

The normalized static stress intensity factor for the same problem but with static load of $4 \times 10^{-3}$ Mbar at both ends of the bar can be estimated with reasonable accuracy, if the maxima and minima of $\tilde{k}_{1}(t)$ are not significantly altered by the dynamic oscillations. We believe that this is the case for our example. The simplest way to obtain $\tilde{k}_{1}$ (statis) is

$$
\begin{aligned}
\tilde{k}_{1}(\text { static }) & =\frac{1}{2}\left[\operatorname{Max} \tilde{k}_{1}(t)-\operatorname{Min} \tilde{k}_{1}(t)\right] \\
& -\left[\operatorname{Min} \tilde{k}_{1}(t)\right] H\left\langle-\operatorname{Min} \tilde{k}_{1}(t)\right\rangle,
\end{aligned}
$$

where $\mathrm{H}$ is the unit Heavislde function, and $\operatorname{Max} f(t)$ and $\operatorname{Min} f(t)$ are the first global maximum and the first giobal min. imum of $f(t)$ respetively. Hence from Fig. 5 and the above formula, $\tilde{\mathrm{k}}_{1}$ (static) $=1.095$, which is about $5.5 \%$ larger than the more accurate theoretical solution, 29 $\tilde{k}_{1}($ static $)=1.038$. Since the dynamic $\tilde{k}_{1}$ has already incorporated an extrapolation, a more accurate method to obtain the static $\tilde{\mathrm{k}}_{1}$ would be to use the calculated $\sigma_{x x}(t)$ a few zones away from the tip. Thus we obtain $k_{1}$ (static) as follows: First obtain $\sigma_{x x}$ (static) from Fig. 8.

$$
\begin{array}{r}
\sigma_{x x}(\text { static })=\frac{1}{2}\left[\operatorname{Max} \sigma_{x x}(t)-\operatorname{Min} \sigma_{x x}(t)\right] \\
-\left[\operatorname{Min} \sigma_{x x}(t)\right] \cdot H\left(-\operatorname{Min} \sigma_{x x}(t)\right) \\
=5.70 \times 10^{-3} \text { Mbar. }
\end{array}
$$

This stress value corresponds to $t_{S}$ $=9.22 \mu \mathrm{sec}$. It is seen in Fig. 5 that for 
$t=t_{s}, \tilde{k}_{1}$ (static) $=1.028$, which is about $1 \%$ less than the value given in Ref. 29. Thus we see that both the dynamic and static values of the stress intensity factor can be calculated with the timedependent HEMP code.

It is important to notice that the dynamic stress intensity factors for a finite crack in an infinite elastic solid are found to be $15-30 \%$ greater than the analogoug static value. ${ }^{13,15}$ These phenomena are attributed to the scattered Rayleigh waves. If there were oniy one incident plane wave in our example, then due to the superposition principle in linear elasticity theory,

$$
\begin{aligned}
& {\left[\operatorname{Max} \tilde{k}_{1}(t)\right]_{\text {one wave }}} \\
& \quad=\frac{1}{2}\left[\operatorname{Max} \tilde{k}_{1}(t)\right]_{\text {two waves }}=1.35 .
\end{aligned}
$$

which is about $30 \%$ larger than the analogous static value. But for the two-wave case, the overshooting is about $160 \%$. This shows that in a practical situation the overshooting of the dynamic stress intensity factor is attributed mainly to

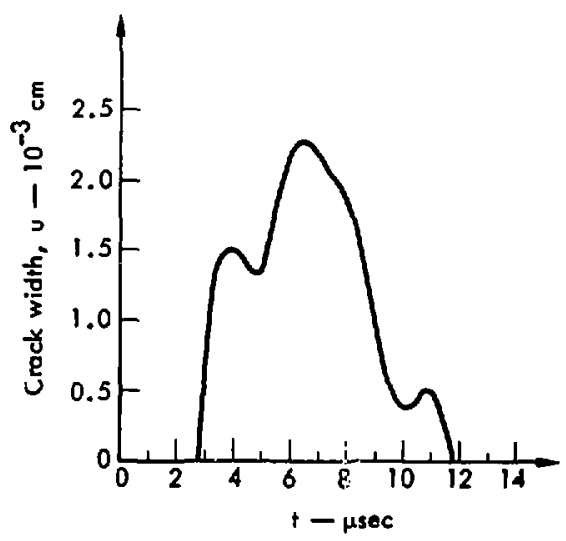

Fig. 9. The u-vs-t curve at the middle of the crack surface.

the particular geometry and the manner of loading.

The geometry of the crack opening and the opening and the closing of crack surfaces as functions of $t$ are the natural products of the HEMP code calculation. They are plotted in Figs, 6 and 9, respectively. In general, the crack opens or close日 when the crack tip experiences tension or compression, respectively.

\section{Acknowledgments}

The author wishes to thank Drs. Mark

L. Wilkins and D. E. Maiden of LLL, and Prof. I. Finnie of the University of California, Berkeley, for many helpful discussions, and Ms. N. Wilt of LLL for programming our problems. 


\section{Appendix \\ Error Analysis}

In the neighborhood of the crack tip, assuming an infinite elastic medium,

$$
\begin{aligned}
& \sigma_{x x}(r, \theta)=k_{1} f_{-1 / 2}(\theta) r^{-1 / 2}+f_{0}(\theta)+f_{1 / 2}(\theta) r^{1 / 2}+f_{1}(\theta) r \\
&+f_{3 / 2}(\theta) r^{3 / 2}+f_{2}(\theta) r^{2}+O\left(r^{5 / 2}\right)
\end{aligned}
$$

where

$$
f_{-1 / 2}(\theta) \neq 0, \text { for } 0 \leq|\theta|<\pi \text {. }
$$

It is well known ${ }^{30}$ that the angular functions $f_{n}(\theta), n=0,1,2, \ldots$, have the sperial property

$$
f_{n}(0)=0, n=0,1,2, \ldots
$$

If we define

$$
k_{1}^{*}(r, \theta)=\frac{r^{1 / 2}}{I_{-1 / 2}(\theta)} a_{x x}(r, \theta),
$$

then $(A-1)$ and $(A-2)$ yield

$$
\begin{aligned}
k_{1}^{* \prime}(r, \theta)=k_{1} \div\left[f_{-1 / 2}(\theta)\right]^{-1} & {\left[f_{0}(\theta) r^{1 / 2}+f_{1 / 2}(\theta) r+f_{1}(\theta) r^{3 / 2}\right.} \\
& \left.+f_{3 / 2}(\theta) r^{2}+f_{2}(\theta) r^{5 / 2}+o\left(r^{3}\right)\right]
\end{aligned}
$$

It is obvious that the best way to obtain higher accuracy is to choose the extrapolation path so that most of the $f^{\prime} s$ vanish. In this particular case, the extrapolation path is along $\theta=0$. From $(A-2)$ and $(A-4)$,

$$
k_{1}^{*}(r, 0)=k_{1}+\left[f_{-1 / 2}(0)\right]^{-1}\left[f_{1 / 2}(0) r+f_{3 / 2}(0) r^{2}+o\left(r^{3}\right)\right]
$$

Hence a straight-line fit of $k_{1}^{*}(r, 0)$ will have an error $O\left(r^{2}\right)$, and a quadratic fit of $k_{1}^{* *}(r, 0)$ will have an error $O\left(r^{3}\right)$. 


\section{References}

1. H. M. Westergaard, "Bearing Pressures and Cracks," J. Appl. Mech., Trans. ASME 61, A-49 (1939).

2. I. N. Sneddon, "The Distribution of Stress in the Neighborhood of a C rack in an Elastic Solid," Pruc. Phys. Soc. London 189, 229 (1946).

3. G. R. Irwin, "Analysis of Stresses and Strains Near the End of a Crack Traversing a Plate." J. Appl. Mech., Trans. ASME 24, 361 (1957).

4. M. L. Williams, "On the Stress Distribution at the Base of a Stationary Crack," J. Appl. Mech., Trans. ASME 24, 309 (1957).

5. B. V. Kostrov, "Motion of a Rigid Strip-Mass Soldered into an Elastic Medium, Excited by a Plane Wave," J. Appl. Math. Mech. 28, 113 (1964).

6. J. F. Loeber and G. C. Sih, "Diffraction of Antiplane Shear Waves by a Finite Crack," J. Acoust. Soc. Amer. 44, 90 (1968).

7. G. C. Sih and J. F. Loeber, "Wave Propagation in an Elastic Solld with a Line of Discontinuity or Finite Crack," Quart. Appl. Math. 27, 193 (1969).

8. J. D. Esinelby, "The Elastic Field of a Crack Extending Nonuniformly Under General Anti-Plane Loading," J. Mech. Phys. Solids 17, 177 (1969).

9. J. D. Achenbach "C rack Propagation Generated by a Horizontally Polarized Shear Wave," J. Mech. Phys. Solids 18, 245 (1970).

10. J. D. Achenbach, "Brittle and Ductile Extension of a Finite Crack by a Horizontally Polarized Shear Wave," Int. J. Eng. Sci. 8 , 947 (1970).

11. A. K. Mal, "Interaction of Elastic Waves with a Penny-Shaped Crack," Int. I. Eng. Sci. $8,381(1970)$.

12. A, K. Mal, "Interaction of Elastic Waves with a Griffith Crack," Int. J. Eng. Sci. 8. $763(1970)$.

13. S. A. Thau and T. H. Lu, "Transient Stress Intensity Factors for a Finite Crack in an Elastic Solid Caused by a Dilatational Wave," Int. J. Solids Structures ? 731 (1971).

14. G. T. Embley and G. C. Sih, "Response of a Penny Shaped Crack to Impact Waves," Proc. 12th Midwestern Merhanics Conference (1971).

15. G. C. Sih, G. T. Embley, and R. S. Ravera, "Impact Response of a Finite Crack in Plane Extension;" Int. J. Solids Structures B, 977 (197z).

16. A. S. Kobayashi, D. E. Maiden, B. J. Simon, and S. Iida, "Application of Finite Element Analysis Method to Two-Dimensional Problems in Fracture Mechanics," ASME Paper No. 69-WA $/$ PVP-12 (1969).

17. S. K. Chan, I. S. Tuba, and W. K. Wilson, "On the Finite Element Method in Tinear Fracture Mechanics," Eng. Fracture Mech. 2, 1 (1970).

18. P. V. Marcal, "Three-Dimensiona: Finite Element Analysis for Fracture Mechanics," The Surface Crack: Physical Problems and Computational Solutions, Ed. by J. L. Swedlow (ASME, New York, 1972), papers presented at the Winter Annual Meeting of ASME. 
19. P. D. Hitton and G. C. Sih, "Application of the Finite Element Mcthod to the Calculation of Stress Intensity Fartors," Mechanics of Fracture I: Methods of Analysis and Solutions of Crack Problems, lid. by G. C. Sih (Noordhoff Int. Pub.. Leyden, The Netherlands, 1973), p. 426.

20. W. K. Wilson, "Finite Element Methods for Elastic Bodies Containing Cracks," Mechanics of Fracture 1: Methods of Analysia and Solutions of Crack Problems. Ed. by G. C. Sih (Noordhoff Int. Pub., Leyden, The Netherlands, 1973), p. 484.

21. E. H. Wu and E. A. Witmer, "Finite F.lement Anelysis of Large Elastic-Plastic Transient Deformations of Simple Structures," ALAA J. 9, 1719 (1971).

22. J. F. McNamara and P. V. Marcal. "Incremental Stiffness Method for Finite Element Analysis of the Nonlinear Dynamic Problem," Proc. Int. Symp. Numerical and Computer Methods in Strurt. Mech., Urbana, IIl., 1971.

23. M. Hartzman and J. R. Hutchinson, "Nonlinear Dymamics of Solids by the Finite Element Methods," Comp. Struct. 2. 47 (1972).

24. Mark L. Wilkins, "Calculation of Elastic-Plastic Flow," Methods in Computational Physics, Ed. by B. Alder, S. Fernbach, and M. Rotenberg (Adademic P'ress, New York and London, 1964), Vol, 3, p. 211-262.

25. Mark L. Wilkins, Calculation of Elastic-Plastic Flow, Lowrence Livermore Laboratory, Rept. UCRL-7322 Rev. 1 (1969); or International Coll, on Gasdynamics of Explosions - Reactive Systems, Moscow, USSR (1969), p. 408.

26. Mark L. Wilkins, S. J. French, and M. Sorem, "Finite Dlfference Scheme for Calculating Problems in Threc Space Dimensions and Time," Proceedings of the Second International Conference on Numerical Methods in Fluid Dynamics, Ed. by M. Holt, Lectur * Notes in Physics Series (Springer-Verlag, New York, 1971). Vol. 8, pp. 30-33.

27. Y. C. Fung, Foundations of Solid Mechanics (Prentice-Hall, Englewood Cliffs, N. J., 1965).

28. T. A. Cruse, "Numerical Evaluation of Elastic Stress Intensity Factors by the Boundary-Integral Equation Method," The Surface Crack: Physical Problems and Computations, Ed. by J. L. Swedlow (ASME. New York, 1072), papers presented at the Winter Annual Mecting of ASME.

29. A. S. Kobayashi, R. D. Cherepy, and W. C. Kinsel, "A Numerical Procedure for Estimating the Stress Intensity Factor of a Crack in a Finite Plate, J. Basic Eng. Trans. ASME, Ser. D. 86, 681 (1964).

30. J. P. Benthem and W. T. Koiter, "Asymptotic Approximntions of Crack Problems," Mechanics of Fracture 1: Methods of Analysis and Solutions of C rack Problems. Ed. by G. C. Sih (Noordhoff Int. Publ., Leyden, The Netherlands, 1973), p. 131. 